Die Nationale Ethikkommission im Bereich der Humanmedizin (NEK-CNE) legt nachstehend ihre Stellungnahme zur Einführung von SwissDRG dar. Ihre Erwägungen entsprechen den Überlegungen, die die FMH als vollwertiger Partner des Projektes von Anfang an gemacht hat. Wir haben immer mit Nachdruck die Wahrung der vollumfänglichen therapeutischen Freiheit verlangt und vor den Grenzen des Wettbewerbs im Gesundheitswesen gewarnt. Im Anschluss an unsere engen Kontakte mit den deutschen Kollegen haben wir uns von An- fang an für eine breitangelegte Forschung über die durch das neue Tarifsystem verursachten Änderungen ausgesprochen. Es ist erfreulich, dass die NEK-CNE als eidgenössische Instanz die Diskussion auf politischer Ebene wieder aufgreift und empfiehlt, das Thema der öffentlichen Diskussion zu unterbreiten.

Dr. med. Pierre-François Cuénoud, Leiter Ressort SwissDRG der FMH

\title{
Zur Einführung von diagnosebezogenen Fallpauschalen in Schweizer Spitälern
}

\begin{abstract}
Einleitung
Tiefgreifende Änderungen stehen den Schweizer Spitälern in den nächsten Jahren bevor. Im Zuge der Revision des Krankenversicherungsgesetzes (KVG) werden bis zum 1. Januar 2012 landesweit für Spitalaufenthalte leistungsbezogene Fallpauschalen eingeführt. Danach soll nicht mehr nach Aufwand oder per Tagespauschalen abgerechnet werden, sondern das Spital erhält pro Patient oder Patientin gemäss einer Diagnose einen fixen Pauschalbetrag (Diagnosebezogene Fallpauschalen, DRG), mit dem es wirtschaften muss. Betroffen sind davon stationäre Behandlungen für somatische Erkrankungen in Spitälern, die über die obligatorische Krankenversicherung abgerechnet werden.
\end{abstract}

Kosten im Gesundheitswesen einzusparen ist aber nicht das primäre Ziel der neuen gesamtschweizerischen Tarifstruktur. Kosteneinsparungen sind auch kaum zu erwarten, da zum einen mit Einführung der DRGs zusätzliche administrative Kosten, zum anderen steigende Kosten im vor- und nachstationären ambulanten Bereich, in der Rehabilitation, in der häuslichen Pflege und in Pflegeinstitutionen anfallen werden. Tragende Argumente für das DRG-System sind vielmehr, dass es eine höhere Transparenz von Kosten ermöglichen sowie Wege eröffnen soll, um die Versorgungsqualität in Spitälern standardisiert messen und deren Leistungen - auch über Kantonsgrenzen hinweg - vergleichen zu können. Weil zukünftig Patientinnen und Patienten ihr Spital selbst wählen und Spitäler wirtschaftlich eigenständiger werden sollen, wird der Wettbewerb zwischen den Spitälern gesteigert werden. Mehr Wettbewerb, so die Hoffnung, zieht eine höhere Effizienz und eine bessere Qualität der klinischen Versorgung nach sich.

Durch die neuen Regeln der finanziellen Abgeltung der Leistungen in Spitälern ergeben sich aber grundsätzlich veränderte Arbeitsvoraussetzungen für die Ärztinnen, Ärzte und Pflegenden, die im Spital tätig sind. Ihr Handlungsspielraum wird zukünftig stärker durch ökonomische Kriterien begrenzt werden. Welche Folgen daraus für die Behandlung der Patientinnen und Patienten erwachsen können, muss sorgfältig überdacht werden, auch aus ethischer Sicht. Mittel- und langfristig sind Veränderungen der funktionalen Rolle und der Leistungen des Spitals sowie ein Wandel der Spitallandschaft zu erwarten. Bis zur flächendeckenden Einführung in der Schweiz ab 2012 vergeht nicht mehr viel Zeit, und eine öffentliche Debatte fehlt bisher. Die Kommission legt hier eine erste Stellungnahme vor, in der sie auf Probleme aufmerksam machen und zu einer konstruktiven Auseinandersetzung bei Gesundheitsfachleuten und in der Öffentlichkeit einladen will.

\section{Hintergrund}

\section{Was sind Fallpauschalen oder DRG?}

DRG («diagnosis related groups») ist ein System, mit dem stationär behandelte Patientinnen und Patienten in medizinisch homogene Gruppen mit ähnlichem Behandlungsaufwand eingeteilt werden. Als Kriterium der Einteilung dient die Hauptdiagnose bei Spitalaustritt, die durch Nebendiagnosen ergänzt werden kann. Jeder Patientenfall kann nur einer einzigen DRG zugeordnet werden. Die Gruppierung und Zuweisung 
einer DRG erfolgt nach einem hierarchisch geordneten Verfahren durch einen «Kodierer».

Das DRG-System kann, wie es bereits in Deutschland konsequent geschieht, als finanzielles Vergütungssystem für Spitäler eingesetzt werden. Der einzelnen DRG kommt je nach Schweregrad der darunter zusammengefassten Krankheitsfälle ein bestimmtes Kostengewicht zu. Die Summe aller Kostengewichte macht den sogenannten Fallmix (Case Mix) aus, dessen Querschnitt (Case-Mix-Index) als Berechnungsgrundlage für die finanzielle Abgeltung von stationären Aufenthalten in einem Spital dient. Die Abgeltung berechnet sich nicht nach der tatsächlich erbrachten Versorgungsleistung und der Verweildauer des Patienten oder der Patientin im Spital, sondern pauschal nach der zugeteilten DRG und deren Kostengewicht. In der Praxis bedeutet dies, dass ein Spital durch die Verkürzung der Verweildauer und durch das Zurückstellen medizinischer und pflegerischer Leistungen Gewinn erzielen oder, falls erbrachte Leistungen das Budget übersteigen, Verluste machen kann.

\section{Der politische Entscheid für SwissDRG}

Das Parlament hat sich 2007 für das KVG-Reformpaket ausgesprochen und eine Spitalfinanzierungsvorlage angenommen, die eine landesweit einheitliche, leistungsbezogene Tarifstruktur für stationäre Spitalaufenthalte vorsieht. Eine Neugestaltung der Tarifstruktur wurde bereits zwischen 2004 und 2007 im Rahmen eines Projektes des Vereins SwissDRG vorbereitet, zu dessen Mitgliedern santésuisse, die Medizinaltarifkommission UVG, MV/IV (MTK), H+ Die Spitäler der Schweiz, die FMH und die Gesundheitsdirektorenkonferenz GDK zählte. Als Beobachter konnten der Schweizer Berufsverband der Pflegefachfrauen und Pflegefachmänner, das Bundesamt für Statistik und das Bundesamt für Gesundheit im Verein Einsitz nehmen. Bereits Ende 2005 entschieden die nationalen Tarifpartner, das aus Deutschland stammende G-DRG-System für die Schweiz zu übernehmen und an die schweizerischen Gegebenheiten anzupassen. Nach dem Entscheid des Gesetzgebers Ende 2007, leistungsbezogene Fallpauschalen bis zum Januar 2012 flächendeckend einzuführen, wurde der Verein SwissDRG Anfang 2008 in eine Aktiengesellschaft mit gemeinnütziger Zweckbindung namens SwissDRG AG überführt, deren Verwaltungsrat sich aus Vertretern der ehemaligen Vereinsmitglieder (nicht der Beobachter) zusammensetzt. Aufgaben der SwissDRG AG sind die Anpassung und Pflege einer neuen Tarifstruktur. Die operative Verantwortung für diese Aufgaben wurde an ein Kompetenzzentrum, das Case Mix Office, delegiert. Der Bund ist weiterhin Beobachter bei den Tarifverhandlungen.

\section{Auswirkungen von diagnosebezogenen Fallpauschalen}

\section{Ökonomisierung des klinischen Alltags}

Therapeutisches Ethos und ökonomische Effizienz müssen sich nicht ausschliessen. Beide Prinzipien sind durch eine Orientierung an einer möglichst effizienten Nutzung vorhandener Mittel miteinander vereinbar - und angesichts der angestrebten gerechten Zuteilung knapper Mittel auch ethisch geboten. Jedoch darf die traditionelle Orientierung des therapeutischen Handelns am Patientenwohl und an der Schadensabwendung nicht durch ein ökonomisches Handlungsprinzip korrumpiert oder gar ersetzt werden. Die Einführung des DRG-Systems verstärkt die Tendenz zu einer weitreichenden Ökonomisierung klinischer Entscheidungen und somit des klinischen Alltags. Dies ist in anderen Ländern (z.B. Deutschland), die mit DRG arbeiten, $\mathrm{zu}$ beobachten. Da ein Spital durch eine günstigere und kürzere Behandlung Gewinn erzielen kann und unter Konkurrenzbedingungen auch erzielen muss, verschieben sich die Interessenkonstellationen zuungunsten der Patientinnen und Patienten. Diese können nicht mehr davon ausgehen, dass therapeutische Massnahmen «in ihrem besten Interesse» ausgewählt und gestaltet werden. Sowohl das ärztliche und pflegerische Handeln als auch der behandelte Patient oder die behandelte Patientin laufen Gefahr, für die Ressourcenökonomie der Klinik instrumentalisiert zu werden.

Auswirkungen sind nicht nur für das ArztPatienten-Verhältnis zu erwarten. Auch die Rolle des Spitals im Schweizer Gesundheitswesen könnte sich im Zuge der DRG-Einführung bedeutend ändern. Zukünftig wird weniger eine breite, patientenorientierte Versorgungsqualität im Vordergrund stehen als vielmehr eine ökonomisch erfolgreiche Betriebsführung des einzelnen Spitals. Auch wird es dem Spital schwerer fallen, soziale Funktionen auszuüben. Sowohl die Ärzteschaft als auch die Pflege wird mit dem Problem konfrontiert werden, dass psychosoziale Leistungen im DRG-System nicht abgebildet und somit auch nicht vergütet werden können. Zeiteinsparungen in der zwischenmenschlichen Kommunikation (auch mit Angehörigen), der Vermittlung von Informationen durch Gespräche und der psychosozialen Begleitung z. B. von Schwerkranken und Sterbenden sind folglich zu erwarten.

Diese Veränderungen widersprechen teilweise dem bisherigen Selbstverständnis der betroffenen Professionen und können zu Spannungen führen zwischen dem therapeutischen Ethos, Hilfe zu leisten, einerseits und der neuen Aufgabe, Krank- 
heitswertigkeiten zu dokumentieren und berechnend ins ärztliche und pflegerische Handeln einfliessen zu lassen, andererseits. Wenn patientenbezogene Entscheidungen verstärkt von ökonomischen Kriterien geleitet werden, die nicht vom Interesse der Patientinnen und Patienten abgeleitet sind, wird unter Umständen gegen das «therapeutische Gewissen» gehandelt werden (müssen), was nicht nur einer optimalen Patientenversorgung widersprechen, sondern auch Frustration und Resignation bei Gesundheitsfachleuten auslösen kann. Aufseiten der Patientinnen und Patienten könnte dieser Zustand in einen Vertrauensverlust gegenüber dem klinischen Personal münden.

\section{Einbussen in der medizinischen und pflegerischen Qualität?}

Durch das DRG-System werden bewusst Anreize geschaffen, Leistungen zu vergünstigen, Massnahmen effizienter oder sparsamer einzusetzen und die Kosten zu reduzieren, die für bestimmte Leistungen entstehen. Das kann neben wünschbaren auch problematische Auswirkungen haben. Aus medizin- und pflegeethischer Sicht sind folgende Fragen zu diskutieren:

- Leistungen vergünstigen bedeutet auch die Verweildauer der Patientinnen und Patienten im Spital zu verkürzen. Da die Schweiz im Vergleich zu anderen europäischen Ländern eine durchschnittlich lange Aufenthaltsdauer in Spitälern vorweist, bedeutet deren Verkürzung nicht grundsätzlich eine Qualitätseinbusse. Entscheidend ist jedoch, dass das DRG-System selbst unzureichende Massnahmen vorsieht, um allzu frühzeitige, medizinisch schwer vertretbare Entlassungen zu vermeiden.

- Eine kürzere Verweildauer kann auch zu steigenden Raten der Rehospitalisierung führen («Drehtüreffekt»).

- Eine Festlegung von Patientinnen und Patienten auf jeweils eine einzige DRG kann verhindern, deren Gesundheitszustand ganzheitlich abzuklären bzw. andere im Laufe des Spitalaufenthalts entdeckte zusätzliche Krankheiten ausreichend zu behandeln. Nach DRG-Logik müsste in einem solchen Fall der Patient oder die Patientin entlassen und erneut mit anderer Hauptdiagnose ins Spital aufgenommen werden. Damit wird eine patientenorientierte therapeutische Behandlung erschwert.

- Zudem können manche Krankheitsfälle und -verläufe eine Komplexität erreichen, die sich schwer oder nur unangemessen in der DRGMatrix abbilden lassen. In solchen Fällen ist eine therapeutisch suboptimale Patientenversorgung zu befürchten.
- Mit der Klassifizierung eines Patienten oder einer Patientin mittels DRG wird nicht en détail festgelegt, welche medizinischen und pflegerischen Leistungen die Person erhalten soll bzw. erhalten hat. Fehlen verbindliche Behandlungsrichtlinien oder Standards, so könnten Einsparungsmassnahmen einer Verminderung der therapeutischen Versorgungsqualität Vorschub leisten.

- Es gibt systemimmanente Anreize zu einem sogenannten «upcoding», d.h. zur Einordnung in eine DRG, die für das Spital mehr Geld abwirft. Das kann sich im Sinn einer Bevorzugung von maximalinvasiven Behandlungsmethoden auswirken, die den besten Interessen der Patientinnen und Patienten zuwiderläuft. Durch die Orientierung am DRGSystem könnte in den Patientenakten zudem ein verzerrtes Krankheitsbild entstehen.

- Ein weiteres Problem ist das Tempo von möglichen Anpassungen des DRG-Systems an medizinische Innovationen. Im Spital wird dies absehbar zu einem Defizit führen, da sich die Innovationen relativ schnell ereignen, während die Fallkosten nur mit Verzögerungen nachgeführt werden können. Zudem sollen Investitionen künftig über die Fallpauschalen durch die öffentlichen Spitäler selbst gedeckt werden - ohne Defizitdeckung seitens der öffentlichen Hand. Dies könnte zu einer Finanzierungslücke führen und damit zu einer Verschärfung der Zweiklassenmedizin.

- Eine Fragmentierung der Behandlung zwischen Spital und günstigeren Nachsorgeeinrichtungen könnte ausserdem zu Verlusten an medizinischer Effizienz führen.

\section{Einbussen in der Verteilungs- und} Zugangsgerechtigkeit im Gesundheitswesen Im DRG-System kann zwischen betriebswirtschaftlich «lukrativen» und «unlukrativen» $\mathrm{Pa}$ tientengruppen unterschieden werden. «Lukrativ» für ein Spital sind Patientinnen und Patienten, die unter dem für die Gruppe festgelegten Aufwand versorgt werden können. «Unlukrativ» sind hingegen diejenigen, deren Behandlungskosten die Pauschale übersteigen. Zur letzteren Gruppe können chronisch Erkrankte, multimorbide Patientinnen und Patienten, geriatrische Patientinnen und Patienten, geistig und körperlich Behinderte sowie Sterbende zählen. Patientinnen und Patienten ohne spezifische Krankheitsdiagnose werden von der DRG-Matrix gar nicht erfasst.

Es ist zu erwarten, dass sich klinische Angebote tendenziell auf «lukrative» Patientengruppen konzentrieren und spezialisieren werden bzw. Spitäler «unlukrative» Patientinnen und 
Patienten frühzeitig entlassen oder in günstigere Übergangspflegeinstitutionen überwiesen werden. Die Tendenz, sich auf «lukrative» Patientengruppen zu konzentrieren, widerspricht dem ethischen Prinzip einer fairen Verteilung von medizinischen Ressourcen. Eine flächendeckende optimale Versorgung müsste daher verstärkt von der öffentlichen Hand sichergestellt werden. Ordnungspolitisch hat der Staat die soziale Verantwortung, auch unter Bedingungen verknappter Ressourcen eine angemessene Versorgungsgerechtigkeit zu garantieren.

Zudem sind Verteilungskämpfe um Pauschalen zwischen verschiedenen Stationen der medizinischen und pflegerischen Versorgung denkbar, wenn ein Spital einen Patienten oder eine Patientin frühzeitig auf Kosten beispielsweise von Spitex oder Rehabilitation entlässt oder an ein anderes Spital überweist. Durch vermehrte Behandlungen im nichtstationären Bereich würden Kosten auf die Patientinnen und Patienten verlagert werden.

Ein weiterer Gerechtigkeitsaspekt wird durch die vergrösserte Eigenverantwortung der Patientinnen und Patienten tangiert. Nicht jede Person ist aus Bildungs-, Alters-, Krankheits- oder anderen Gründen in der Lage, sich über die Spitalleistungen zu informieren und eine entsprechende Spitalwahl zu treffen. Da es sich bei Erkrankten aus ethischer Perspektive immer um eine vulnerable Personengruppe handelt, die eines besonderen Schutzes bedarf, ist die Übertragung des Modells eines aufgeklärten und rational entscheidenden «Konsumenten» auf den klinischen Kontext zu hinterfragen.

\section{Datenschutz}

Zur Abrechnung von Spitalleistungen mit DRGSystem müssen unter Umständen detaillierte Informationen über Diagnosen an die Versicherer weiter gegeben werden. Es wäre aber fraglich, wie unter diesen Umständen das Patientengeheimnis effektiv aufrechterhalten werden kann. Eine Übermittlung von vertraulichen Patientendaten an die Versicherer könnte einen Eingriff in die Persönlichkeitsrechte der Patientinnen und Patienten darstellen.

\section{Folgerungen und Empfehlungen}

Die Einführung leistungsbezogener Fallpauschalen ist eine Entscheidung des Gesetzgebers. Jedoch besteht bei der konkreten Ausgestaltung und Umsetzung des neuen Systems mit der angestrebten «Helvetisierung» des aus Deutschland übernommenen DRG-Systems Gestaltungsspielraum. Mögliche negative Tendenzen wie Einbussen in der medizinischen, pflegerischen und psychosozialen Versorgungsqualität in Spitälern, der Verlust sozialmedizinischer Funktionen des
Spitals, der Verlust einer patientenzentrierten Behandlung, die Benachteiligung bestimmter Patientengruppen und der gefährdete Datenschutz könnten bereits im Vorfeld erkannt, teilweise abgewendet oder mit flankierenden Massnahmen versehen und dadurch abgemildert werden. Der Bund muss seine leitende Verantwortung bei der Qualitätssicherung wahrnehmen.

Die NEK-CNE leitet daraus folgende Empfehlungen ab:

1. Über das DRG-System ist eine offene Debatte über die positiven, aber auch über mögliche nachteilige Effekte zu führen, und es gilt, rechtzeitig über die Massnahmen zur Behebung negativer Folgen nachzudenken.

2. Diese Debatte muss über die Fachkreise hinaus in die Öffentlichkeit getragen werden. Denn die tiefgreifenden Umwälzungen, die durch die Einführung des DRG-Systems zu erwarten sind, sind der breiten Bevölkerung bislang noch weitgehend unbekannt.

3. Die Erfahrungen aus anderen Ländern sollen ausgewertet werden, weil sie hilfreiche Informationen liefern könnten.

4. Zur Einführung des Systems ist es wichtig, das Spitalpersonal (inklusive Ärzteschaft und Pflege) ausreichend zu informieren und auszubilden.

5. Für eine aussagekräftige Qualitätskontrolle ist es dringend nötig, rechtzeitig eine ausreichend breitangelegte wissenschaftliche, von verschiedenen Disziplinen ausgeführte Begleitforschung einzurichten. Um einen Vergleich mit dem Zustand vor der Umstellung auf das neue Vergütungssystem zu ermöglichen (Einführungseffekte), muss die Begleitforschung bereits vor der Einführung des Systems beginnen. Die Begleitforschung muss unabhängig sein und finanziell genügend alimentiert werden. $\mathrm{Zu}$ ihren Fragestellungen sollte es gehören, die Auswirkungen des DRG-Systems auf die Qualität der klinischen Versorgung, auf deren Kosten und vor allem auf die Gesundheitszustände der verschiedenen Patientengruppen aufzuzeigen. Veränderungen der Patientenflüsse in und zwischen verschiedenen Leistungserbringern sollten ebenso erfasst werden wie die Charakteristika von Patientinnen und Patienten in einzelnen Institutionen. Die Forschung sollte aber auch qualitativ und quantitativ festhalten, wie sich die Arbeitssituation für die verschiedenen Berufsgruppen im Gesundheitswesen verändert, insbesondere für Ärzteschaft und Pflegende. Solche Veränderungen haben möglicherweise eine direkte Auswirkung auf die Qualität einer umfassenden Versorgung von Erkrankten. 